\title{
Magnetic anisotropy in strained manganite films and bicrystal junctions
}

\author{
V. V. Demidov, ${ }^{1, \text { a) }}$ G. A. Ovsyannikov, ${ }^{1,2}$ A. M. Petrzhik, ${ }^{1}$ I. V. Borisenko, ${ }^{1}$ A. V. Shadrin,,${ }^{1,2}$ \\ and R. Gunnarsson ${ }^{2,3}$ \\ ${ }^{1}$ Kotel'nikov Institute of Radio Engineering and Electronics Russian Academy of Sciences, \\ 125009 Moscow Mokhovaya 11, Russia \\ ${ }^{2}$ Chalmers University of Technology, S-41296 Gothenburg, Sweden \\ ${ }^{3}$ Jonkoping University, S-55111 Jonkoping, Sweden
}

(Received 16 November 2012; accepted 4 April 2013; published online 29 April 2013)

Transport and magnetic properties of $\mathrm{La}_{0.67} \mathrm{Sr}_{0.33} \mathrm{MnO}_{3}$ (LSMO) manganite thin films and bicrystal junctions were investigated. Epitaxial manganite films were grown on $\mathrm{SrTiO}_{3}, \mathrm{LaAlO}_{3}, \mathrm{NdGaO}_{3}$ $(\mathrm{NGO})$, and $\left(\mathrm{LaAlO}_{3}\right)_{0.3}+\left(\mathrm{Sr}_{2} \mathrm{AlTaO}_{6}\right)_{0.7}$ substrates, and their magnetic anisotropy were determined by two independent techniques of magnetic resonance spectroscopy. It was demonstrated that by using these techniques, a small (0.3\%) anisotropy of crystal structure at the (110) surface plane of the orthorhombic NGO substrate leads to uniaxial magnetic anisotropy of the films in the plane of the substrate at least at the room temperature. It was found that on vicinal NGO substrates, the value of magnetic anisotropy strength can be varied in the range 100-200 Oe at $\mathrm{T}=295 \mathrm{~K}$ by changing the substrate vicinal angle from $0^{\circ}$ to $25^{\circ}$. Measurement of the magnetic anisotropy of manganite bicrystal junction demonstrated the presence of two ferromagnetic spin subsystems for both types of bicrystal boundaries with tilting of basal plane of manganite tilted bicrystal (TB-junction) and with rotation of crystallographic axes (RB-junction) used for comparison. The magnetoresistance of TB-junctions increases with decreasing temperature and the misorientation angle. Variation of bicrystal misorientation angle does not lead to change of misorientation of easy magnetic axes in the film parts forming TB-junction. Analysis of the voltage dependencies of bicrystal junction conductivity show that the low value of the magnetoresistance for the LSMO bicrystal junctions can be caused by two scattering mechanisms. The first one is the spin-flip of spin-polarized carriers due to the strong electron-electron interactions in a disordered layer at the bicrystal boundary at low temperatures and the second one is spin-flip by antiferromagnetic magnons at high temperatures. (C) 2013 AIP Publishing LLC.

[http://dx.doi.org/10.1063/1.4802659]

\section{INTRODUCTION}

Ferromagnetic materials where the spin polarization of carriers is close to $100 \%$ are attractive for use in basic elements of spintronic devices, particularly in magnetic junctions, where the manipulations are made not with charge but with the spin state of the system. ${ }^{1-5}$ Rare-earth manganite perovskites of the type $\mathrm{Re}_{1-\mathrm{x}} \mathrm{A}_{\mathrm{x}} \mathrm{MnO}_{3}$ (where Re is a rareearth element like $\mathrm{La}$ or $\mathrm{Nd}$ and $\mathrm{A}$ is an alkaline-earth metal like $\mathrm{Sr}$ or $\mathrm{Ca}$ ) exhibit a wide spectrum of unusual electrical and magnetic properties, including nearly $100 \%$ spin polarization and the colossal magnetoresistance (CMR) effect (see reviews Refs. 1-4). In the manganite based magnetic junctions, the record values of the magnetoresistance were demonstrated and hence new strong effects caused by highly spin-polarized injection could be expected (see, e.g., Ref. 6).

The properties of epitaxial manganite films used for fabrication of magnetic junctions may differ substantially from the properties of single crystals. As it was shown earlier, ${ }^{2,3,7-14}$ the strain, arising in the epitaxial films due to the mismatch with the substrate, is responsible for this difference. It was demonstrated that the three-dimensional compression of the manganite crystal lattice increases the

a)e-mail: demidov@ cplire.ru hopping probability amplitude within the double-exchange model, which results in an increase of the Curie temperature $T_{\mathrm{C}}{ }^{14}$ whereas biaxial distortions of the Jahn-Teller type lead to an enhancement of electron localization and to a decrease of the Curie temperature. ${ }^{7,8,13}$ The magnetic properties of manganite films can be substantially affected by the phase separation phenomena and the presence of a nonmagnetic layer at the substrate-film interface. ${ }^{11}$ However, a number of problems associated with the influence of the strain on the magnetic properties of manganite films and magnetic junctions $s^{3,4,7,8}$ as well as the influence of the substrate crystallographic symmetry on the magnetic anisotropy and Curie temperature $^{7,15}$ have remained unclear and require further investigation.

It was found that in some manganites apart from the cubic magnetic anisotropy induced by the crystal structure of manganites, thin films exhibit a uniaxial in-plane anisotropy which is significantly stronger than the cubic one at least at room temperature. ${ }^{15-19}$ The uniaxial anisotropy is assumed to be provoked by mechanical strain caused by the misfit between the lattice parameters of the film and substrate materials. The growth and magnetic properties of epitaxial $\mathrm{La}_{1-\mathrm{x}} \mathrm{Sr}_{\mathrm{x}} \mathrm{MnO}_{3}$ (LSMO) films was studied in Ref. 15 for the (110), (001), (100), and (010) orientated $\mathrm{NdGaO}_{3}$ (NGO) substrate. For all NGO substrate orientations, in-plane 
uniaxial magnetic anisotropy was observed at all temperatures up to $T_{\mathrm{C}}$, which also was explained by misfit induced stresses in the film. In the case of (001) $\mathrm{SrTiO}_{3}$ (STO) substrates, an in-plane cubic anisotropy is typically observed. However, when the substrate surface is cut so that there is a small angle $\left(0.13^{\circ}, 0.24^{\circ}\right)$ between the [001] direction and the normal of the substrate surface, a uniaxial anisotropy in the substrate plane was observed at room temperature. A predominant biaxial anisotropy was observed at liquid nitrogen temperatures in LSMO films deposited on a (001) $\mathrm{SrTiO}_{3}$ substrate in which the (001) plane was tilted at an angle of $10^{\circ}$ with respect to the substrate surface. ${ }^{19}$

The biaxial and uniaxial magnetic anisotropy in epitaxial (001)-oriented $\mathrm{LSMO}$ and $\mathrm{L}_{0,67} \mathrm{Ca}_{0,3} \mathrm{MnO}_{3}$ (LCMO) films on substrates of STO, $\mathrm{LaAlO}_{3}(\mathrm{LAO}), \mathrm{MgO}$, and buffered $\mathrm{Si}$ ( $\mathrm{Si} / \mathrm{YSZ}$ ) were measured by the torque magnetometry in the range from $T=20 \mathrm{~K}$ to the room temperature in Ref. 18. It was demonstrated that the cubic magnetic in-plane anisotropy decreased strongly with increasing temperature, while a uniaxial one stays constant or slightly decrease for these substrates. Later, the increase of uniaxial magnetic out-of-plane anisotropy with decreasing temperature was also shown for LSMO/LAO films. ${ }^{18}$

Besides the study of the magnetic anisotropy in manganite films, the magnetic junctions have been the subject of intense research also. ${ }^{20-25}$ The fabrication of the manganite magnetic junctions is complicated because of their high sensitivity to both the degradation of the chemical composition and the change of the electronic states near the interface. One way to obtain the magnetic junction is to create a bicrystal boundary in thin epitaxial films by epitaxial growth of the film on a substrate consisting of two misoriented single crystal pieces. Much attention has been paid to study the manganite bicrystal junctions at the boundaries obtained in epitaxial films grown on STO bicrystal substrates with a rotation of the crystallographic axes of the manganites around the normal to the plane of the substrate (Rotated Bicrystal Junction-RB-junction). ${ }^{20-24}$ The resulting junctions exhibited of several tens of percent tunnel magnetoresistance at fields below $1 \mathrm{kOe}$ and its characteristic resistance varies in a wide range depending on the quality of bicrystal substrate boundary $\left(10^{-7}-10^{-5} \Omega \cdot \mathrm{cm}^{2}\right)$. For example, it was demonstrated that tunnel magnetoresistance was increased with increasing of the misorientation angle from $0^{\circ}$ to $45^{\circ} .{ }^{24}$ The CMR and the anisotropic magnetoresistance (AMR) of the junction can be neglected at such a high value of tunneling magnetoresistance.

Previous studies of bicrystal junction made of cuprate superconductors $^{26,27}$ showed that the microstructure of the boundary of the junction formed by rotation of crystallographic planes around the bicrystal boundary is significantly improved as compared with RB-junction boundary. This type of bicrystal boundaries has a low density of dislocations in the boundary plane and has a better morphology of the boundary. ${ }^{26}$ The first experiments carried out on $\mathrm{L}_{0,67} \mathrm{Ca}_{0,3} \mathrm{MnO}_{3}$ Tilted Bicrystal (TB-junction) showed high values of TMR $(150 \%)$ with a rather large value of the resistance of bicrystal boundaries- $-(3-5) \cdot 10^{-5} \Omega \cdot \mathrm{cm}^{2}$ (Refs. 27 and 28). At the same time, the magnetoresistance in
$\mathrm{La}_{1-\mathrm{x}} \mathrm{Sr}_{\mathrm{x}} \mathrm{MnO}_{3}$ TB-junction was found to be only a few percents and was comparable with the AMR contribution of manganite films. ${ }^{28}$

The aim of this work is to study the magnetic and transport properties of strained manganite films and manganite bicrystal junctions. We mainly concentrate on the LSMO film and bicrystal junction prepared on NGO substrate because the magnetic in-plane uniaxial anisotropy is more favourable for observation of higher magnetoresistance. Other substrates like STO, LAO, and $\left(\mathrm{LaAlO}_{3}\right)_{0.3}+\left(\mathrm{Sr}_{2} \mathrm{AlTaO}_{6}\right)_{0.7}$ (LSAT) were studied as reference. Section II presents the technique of fabrication and experimental methods we used in our study. In Sec. III, we discuss the magnetic anisotropy of thin LSMO films and present the results of measurements of magnetic parameters in bicrystal junctions. Section IV presents the results of measurements of transport parameters of the bicrystal junctions. A comparison of the parameters of bicrystal RB-junctions with in-plane misoriented axes with bicrystal TB-junctions is made. The contributions of the colossal magnetoresistance and anisotropic magnetoresistance of the films in bicrystal junctions are estimated. Section V provides conclusions of the work.

\section{SAMPLE FABRICATION AND EXPERIMENTAL TECHNIQUE}

\section{A. Thin film growth and characterization}

The epitaxial films of LSMO and LCMO with thickness of $50-70 \mathrm{~nm}$ were grown by pulsed laser ablation at 750 $800^{\circ} \mathrm{C}$ and oxygen pressure $0.2-0.3$ mbar. ${ }^{30}$ We fabricated two sets of the films. First, we grew the films on tilted substrates to obtain the controllable mechanical strain in the films for the magnetic anisotropy study. Second, we grew the films on the bicrystal substrates to study bicrystal magnetic junctions. Most of the films were deposited on NGO substrates, in which the crystallographic plane (110) NGO was rotated around the [001] NGO by several fixed angles varying from $0^{\circ}$ to $26^{\circ}$. We use orthorhombic nomination for NGO substrate as in Ref. 15. Moreover, we neglect orthorhombicity for LSMO and LCMO and consider it as a cubic material. ${ }^{2-4}$ To illustrate the effects of anisotropy induced by the substrate material, we also used several LSMO films, deposited on (001) LSAT, (001) LAO, and (001) STO substrates. The bicrystal NGO substrates with the symmetric rotation of (110) NGO planes around [11̄0] NGO by the angles $2 \theta=12^{\circ}, 22^{\circ}, 28^{\circ}$, and $38^{\circ}$ (see Fig. 1) were used for fabrication of the TB-junctions. RB-junction with misorientation angle $2 \theta^{\prime} \approx 90^{\circ}$ were prepared on the substrate where the axes of (110) NGO plane are rotated around the normal to the substrate. The crystallographic parameters of the films and substrates were characterized using a 4-circle X-ray diffractometer. ${ }^{30}$

The manganite films grown on NGO substrates have the same epitaxial relationship for both LSMO and LCMO films. We have the following relations: (001) LSMO//(110) NGO and [100] LSMO//[110] NGO for the LSMO films. The pseudocubic lattice constant for LSMO $a_{\mathrm{LSMO}}=0.388 \mathrm{~nm}$ (for $\mathrm{LCMO} a_{\mathrm{LCMO}}=0.3858 \mathrm{~nm}$ ), while the lattice constants of (110) NGO substrate (orthorhombic cell $a=0.5426 \mathrm{~nm}$, 


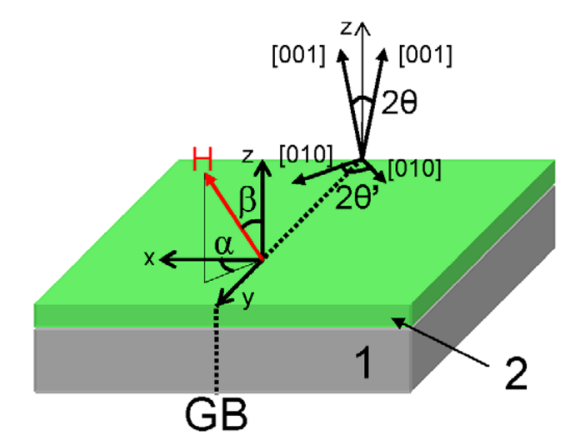

a

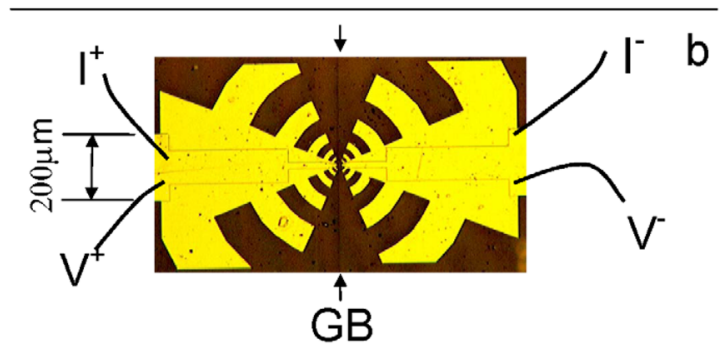

FIG. 1. (a) Schematic view of the bicrystal boundary (GB) in manganite thin film (2) deposited on bicrystal substrate (1). The crystallographic directions of bicrystal configuration for two parts of the (001) LSMO film are indicated by arrows. The misorientation angles for RB and TB boundary are marked by $2 \theta^{\prime}$ and $2 \theta$, respectively. Angles and the direction of magnetic field $\mathrm{H}$ are determined by the polar angle $\alpha$ and the azimuthal angle $\beta$. Axis $x$ corresponds to current flow direction and $y$ is along the bicrystal boundary. (b) A photo of a bicrystal junction connected with logoperiodic antenna.

$b=0.5502 \mathrm{~nm}$, and $c=0.7706 \mathrm{~nm}$ ) along the [001] and [1ํㅣㄹ directions are equal to $a_{[001]}=0.3853 \mathrm{~nm}$ and $b_{[1 \overline{1} 0]}$ $=0.3863 \mathrm{~nm}$ accordingly. ${ }^{30,31} \mathrm{We}$ found by means of X-ray diffractometry that the above mentioned epitaxial condition for LSMO films are valid at least for miscut angles up to $28^{\circ}$ for the substrates with tilted (110) NGO plane. The following strain relations take place for (001) LCMO films having smaller lattice parameters $\left(a_{[001]}<a_{L C M O}<b_{[1 \overline{1} 0]}\right)$ : a compression along the [001] NGO and a tension along the [1 $\overline{1} 0]$ NGO. For (001) LSMO film deposited on (110) NGO substrate, the strain is compressive for both directions.

Bridges with a width of $6-8 \mu \mathrm{m}$ crossing the bicrystal boundary were formed by ion-beam etching using a photoresist mask (Fig. 1). All transport measurements were made by using the four-point method with platinum or gold contact pads. DC current flowing in the film plane was perpendicular to the boundary, and the direction of the external magnetic field was determined by two angles: the polar $\alpha$ and azimuthal $\beta$ (Fig. 1(a)).

\section{B. Resonance microwave technique}

Two independent methods, based on ferromagnetic resonance absorption of electromagnetic radiation in the films, were applied to determine the parameters of the magnetic anisotropy. We used the electron spin resonance (ESR) spectrometer ER-200 Bruker (frequency 9.61 GHz) to obtain the angular dependence of ESR spectra. The DC magnetic field and the magnetic component of the RF field were directed perpendicular to each other and remained in the plane of the film during the rotation (so-called "the parallel configuration"). The rotation was performed around an axis perpendicular to the substrate plane. This technique eliminates the change in signal due to thin film shape anisotropy and allows us to measure the in-plane magnetic anisotropy only. The relation between the frequency of the electromagnetic radiation and the resonance magnetic field $H_{0}$ of the FMR can be expressed in analytical form ${ }^{31}$

$$
\begin{aligned}
\left(\frac{\omega}{\gamma}\right)^{2}= & \left(4 \pi M_{0}+H_{0}+\frac{2 K_{u}}{M_{0}} \cos ^{2} \varphi_{u}+\frac{2 K_{c}}{M_{0}} \frac{1+\cos ^{2} 2 \varphi_{c}}{2}\right) \\
& \times\left(H_{0}+\frac{2 K_{u}}{M_{0}} \cos 2 \varphi_{u}+\frac{2 K_{c}}{M_{0}} \cos 4 \varphi_{c}\right)
\end{aligned}
$$

where $\omega$ is the angular frequency, $\gamma$ is the gyromagnetic ratio, $M_{0}$ is the equilibrium magnetization per unit volume, $\varphi_{u}$ and $\varphi_{c}$ are the angles between the external magnetic field and the in-plane easy axes of uniaxial and cubic anisotropy, respectively, and $K_{u}$ and $K_{c}$ determine the uniaxial anisotropy field $H_{u}=2 K_{u} / M_{0}$ and the cubic anisotropy field $H_{c}=2 K_{c} / M_{0}$, correspondingly. ${ }^{31}$

The spin-dependent transport in the bicrystal junctions uses a much smaller external magnetic fields than one is required to observe the FMR spectra at X-band (DC magnetic field is around $3 \mathrm{kOe}$ ). Therefore, we used the second method in the work that was based on the significant increase of the static magnetic susceptibility $\chi_{0}$ in a ferromagnetic with uniaxial anisotropy. In this case, the external magnetic field should be oriented along the hard axis and varied in vicinity of the uniaxial anisotropy field value. The field dependence of the static magnetic susceptibility in uniaxial ferromagnetic films has a sharp peak in vicinity of $H_{\mathrm{u}}$, when the external field is directed along the hard axis of magnetization. ${ }^{32,33}$ Consequently, it is possible to obtain a direction and a value of the uniaxial anisotropy field by recording the sharp peak of RF absorption that is proportional to the imaginary magnetic susceptibility which in turn is proportional to the static magnetic susceptibility.

To implement the second method, a magnetic resonance spectrometer operating on the basis of Q-meter at the frequency $300 \mathrm{MHz}$ was used. ${ }^{34}$ The DC magnetic field dependence of the absorption spectra is measured for magnetic field orientation near the hard axis of magnetization. An external DC magnetic field is varied in the range from -300 Oe to +300 Oe. The sharp increase of the absorption signal indicates that DC magnetic field is equal to the value of the uniaxial anisotropy field.

\section{MAGNETIC ANISOTROPY IN MANGANITE FILMS AND BICRYSTALS}

\section{A. Strain in manganite film}

The interplane distance $a_{\perp}$ of the LSMO films taken along the direction [001] LSMO and the lattice constant $a_{s}$ of the substrate were determined by using the X-ray diffraction technique. Figure 2 shows $2 \theta-\omega$ scans in the vicinity of the (002) reflections of LSMO film deposited on (001) LAO, (110) NGO, and (001) STO substrates. It can be seen from Fig. 2 that the interplane distance of the LSMO film $a_{\perp}$ strongly depends on the substrate lattice parameter $a_{s}$. The lattice constant $a_{\perp}$ (see inset Fig. 2) of LSMO film were 


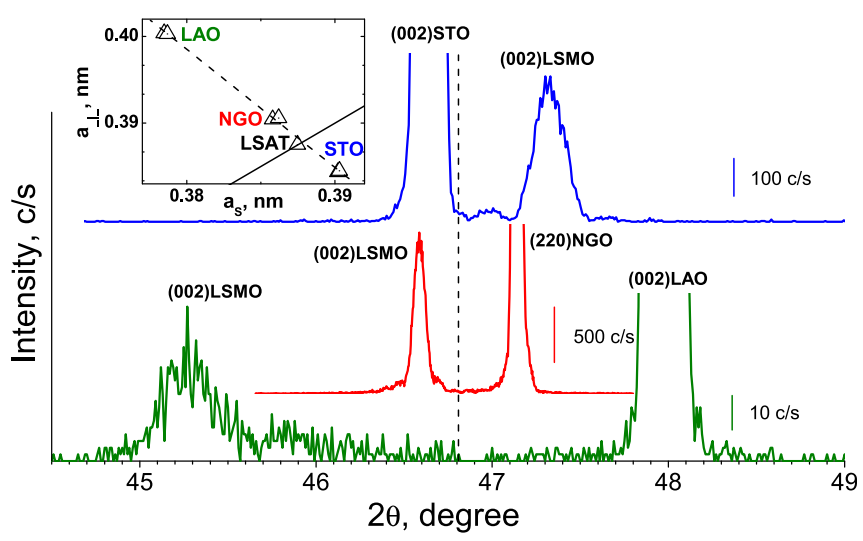

FIG. 2. X-ray diffraction patterns (measured in the $2 \theta / \omega$ scan mode, log scale on intensity) of the LSMO films deposited onto LAO, NGO, and STO substrates. The dashed line indicates the position of the hypothetical reflection for bulk (002) LSMO. ${ }^{14}$ Inset: the dependence of interplane distance of LSMO films $a_{\perp}$ (triangular) on $a_{\mathrm{s}}$ is shown. Solid line is the dependence of $a_{\perp}=a_{\mathrm{s}}$, which is true for unstrained cubic lattice.

determined from the several $(00 n)$ peaks $(n=1,2,3)$ of the LSMO films and substrates. The intersection point of the dependence $a_{\perp}\left(a_{s}\right)$, which is linear for small mismatch with the straight line $a_{\perp}=a_{s}$, gives us the lattice constant of unstrained LSMO film $a_{\mathrm{LSMO}}=0.387 \pm 0.014 \mathrm{~nm}$. This value coincides with the results obtained in Ref. 14 for polycrystalline samples. Consequently, we can conclude that our manganite films are fully strained without any sign of relaxation within the experimental errors. These films are compressively strained for the NGO and LAO substrates and tensely strained for STO and LSAT substrates.

Table I presents the lattice parameters $a_{\perp}$ in the direction [001] LSMO films deposited onto NGO substrates in which the (110) plane is tilted by the angles $\theta_{1}$ from $0^{\circ}$ to $25.7^{\circ}$ around the $[1 \overline{1} 0]$ NGO direction. Rocking curve widths $\Delta \omega$ of LSMO films are also presented in Table I. The parameters of LSMO films deposited onto STO and LSAT substrates are given for comparison.

The LSMO films grown on the tilted NGO substrates are oriented with the same epitaxial relation (001) LSMO//(110) $\mathrm{NGO}$ and [100] LSMO//[110] NGO. So for the film

TABLE I. Lattice constant and rocking curve widths for LSMO films deposited on NGO substrate with varying miscut from the (110) plane.

\begin{tabular}{lccc}
\hline \hline Substrate orientation $^{\mathrm{a}}$ & $\theta_{1}(\mathrm{deg})^{\mathrm{b}}$ & $a_{\perp}(\mathrm{nm})^{\mathrm{c}}$ & $\Delta \omega(\mathrm{deg})^{\mathrm{d}}$ \\
\hline$(110)$ NGO & 0 & 0.3904 & 0.037 \\
$(450)$ NGO & 6 & 0.3904 & 0.04 \\
$(230) \mathrm{NGO}$ & 10.9 & 0.3916 & 0.08 \\
$(120)$ NGO & 18.7 & 0.3913 & 0.05 \\
$(130)$ NGO & 25.7 & 0.3912 & 0.08 \\
$(001) \mathrm{STO}$ & 0 & 0.3845 & 0.014 \\
$(001) \mathrm{LSAT}$ & 0 & 0.3875 & 0.06 \\
\hline \hline
\end{tabular}

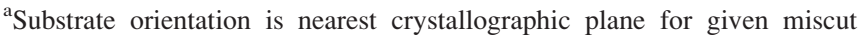
angle from the plane (110).

${ }^{\mathrm{b}} \theta_{l}$ is the miscut angle.

${ }^{\mathrm{c}} a_{\perp}$ is the lattice parameters in c-direction.

${ }^{\mathrm{d}} \Delta \omega$ is the FWHM of rocking curve. Parameters for the films deposited on (001) STO and (001) LSAT substrates are shown for comparison. deposited on tilted substrate LSMO film octahedral rotation occurs. The lattice constant of the film $\mathrm{a}_{\perp}$ is constant at $\theta_{1}=0^{\circ}$ and $\theta_{1}=6^{\circ}$, then it increases to maximum at $\theta_{1}=11^{\circ}$ to decrease slowly with increasing $\theta_{1}$. Here, we take these experimental results "as is" and leave its explanation for the future. We do not think that any crystallographic symmetry changes LSMO influences on the lattice constant of the film.

\section{B. Magnetic anisotropy}

Now, we present our experimental results on magnetic anisotropy of manganite films obtained by FMR spectroscopy at the room temperature. It is interesting to note that we have found a biaxial magnetic anisotropy induced by the cubic structure of LSMO in all films under study. Figure 3 shows an example of the angular dependence of the resonance field of the FMR line $\mathrm{H}_{0}$ that was measured at frequency of 9.61 $\mathrm{GHz}$ for LSMO films deposited on NGO, LSAT, and STO substrates. It should be noted the minimum of the resonance field corresponds to the DC magnetic field direction along the easy axis. Angle of rotation $\varphi$ was measured from the [110] NGO direction, which was detected as a magnetization easy axis of the LSMO film, ${ }^{15}$ and $\varphi=\pi / 2-\alpha$ (see Fig. 1(a) for TB-boundary only). It is clearly seen that the contribution of uniaxial anisotropy in the case of LSMO/NGO film (see circles in Fig. 3) is substantially larger than the contribution of the cubic anisotropy which is clearly seen for LSMO/ LSAT film. For LSMO/NGO film, angles of $0^{\circ}$ and $180^{\circ}$ correspond to the easy axis and angles of $90^{\circ}$ and $270^{\circ}$ correspond to the hard axis. For LSMO/STO film, a uniaxial anisotropy is not so pronounced (see Fig. 3); nevertheless, detail analysis of the experimental data shows its presence in the films. ${ }^{31}$ The angle dependences for LSMO/LSAT and LSMO/STO films were fitted by a horizontal displacement for a convenient comparison with LSMO/NGO data.

Table II gives the parameters for the seven different tilt angles of LSMO/NGO films that were obtained from analysis of the FMR angular experiments by using Eq. (1). Our experimental data of the angle dependences were fitted by Eq. (1) with the five fitting parameters: $\mathrm{M}_{0}, \mathrm{H}_{\mathrm{u}}, \mathrm{H}_{\mathrm{c}}, \varphi_{\mathrm{u}}$, and $\varphi_{\mathrm{c}}$. It should be noted that the high sensitivity of ESR spectrometer allows us to determine all these five parameters of

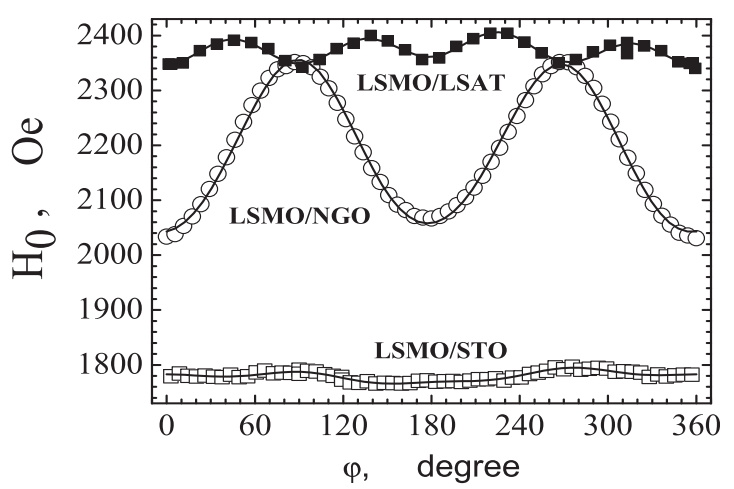

FIG. 3. Angular dependences of the FMR field $\mathrm{H}_{0}$ for (001) LSMO film in LSMO/LSAT, LSMO/NGO, and LSMO/STO measured at frequency of 9.61 $\mathrm{GHz}$ and $\mathrm{T}=300 \mathrm{~K}$. Here, symbols are experimental data and solid lines are the calculation using Eq. (1). 
TABLE II. Parameters of magnetic anisotropy for LSMO films deposited on NGO substrate with varying miscut from the (110) plane.

\begin{tabular}{lccccc}
\hline \hline$\theta_{1}(\mathrm{deg})^{\mathrm{a}}$ & $H_{\mathrm{u}}(\mathrm{Oe})^{\mathrm{b}}$ & $H_{\mathrm{c}}(\mathrm{Oe})^{\mathrm{c}}$ & $\Delta \varphi_{\mathrm{c}}(\mathrm{deg})^{\mathrm{d}}$ & $K_{\mathrm{u}}\left(\mathrm{kErg} / \mathrm{cm}^{3}\right)^{\mathrm{e}}$ & $K_{\mathrm{c}}\left(\mathrm{kErg} / \mathrm{cm}^{3}\right)^{\mathrm{e}}$ \\
\hline 0 & 105 & 13.6 & 45.7 & 17.5 & 2.25 \\
6.0 & 153 & 14 & 42.5 & 21.4 & 1.96 \\
6.5 & 125 & 10.4 & 45.6 & 11.1 & 0.93 \\
11 & 86 & 6.7 & 45 & 10.9 & 0.85 \\
18.7 & 122 & 15 & 46 & 13.8 & 1.70 \\
21 & 158 & 14.9 & 43.6 & 23.2 & 2.20 \\
25.7 & 197 & 20 & 43.6 & 31.7 & 3.22 \\
\hline \hline
\end{tabular}

${ }^{\mathrm{a}} \theta_{1}$ is the miscut angle.

${ }^{\mathrm{b}} H_{\mathrm{u}}$ is the uniaxial anisotropy field.

${ }^{\mathrm{c}} H_{\mathrm{c}}$ is the cubic anisotropy field.

${ }^{\mathrm{d}} \Delta \varphi_{\mathrm{c}}$ is the angle between easy axes for cubic and uniaxial anisotropy.

${ }^{\mathrm{e}} K_{u}$ and $K_{c}$ are the constants of uniaxial and cubic anisotropy, respectively.

magnetic anisotropy with accuracy to within a few percent. It is seen from Table II that all films deposited on NGO substrate have biaxial anisotropy induced by the cubic structure of LSMO. At room temperature, this anisotropy is at least one order of magnitude lower than the uniaxial anisotropy induced by the strain. The angles between the easy axes of these two types of anisotropy $\Delta \varphi_{c}$ are close to $45^{\circ}$ for all samples.

Figure 4 shows the experimental values of uniaxial anisotropy constant $K_{u}$ for LSMO films with various (110) NGO plane tilt angles $\theta_{1}$. The anisotropy constants were calculated using $K_{u}=H_{u} M_{0} / 2$, where the values of $H_{u}$ were determined independently from the two types of experiments: measurements of the angular dependences of FMR spectra at a frequency of $9.61 \mathrm{GHz}$ and the angular dependence of the absorption spectra at a frequency of $290.6 \mathrm{MHz}$.

Let us consider how the film anisotropy is related to the additional mechanical strains induced by the tilting of (110) NGO plane. We can write the following expression for the free energy density of a ferromagnetic sample without taking into account the formation of a domain structure at a tilted epitaxial film growth plane and assuming nothing about the character anisotropy: ${ }^{35}$

$$
F=-(\mathbf{M} \cdot \mathbf{H})+\frac{1}{2}(\mathbf{M} \cdot \hat{N} \cdot \mathbf{M})+F_{\mathrm{mc}} .
$$

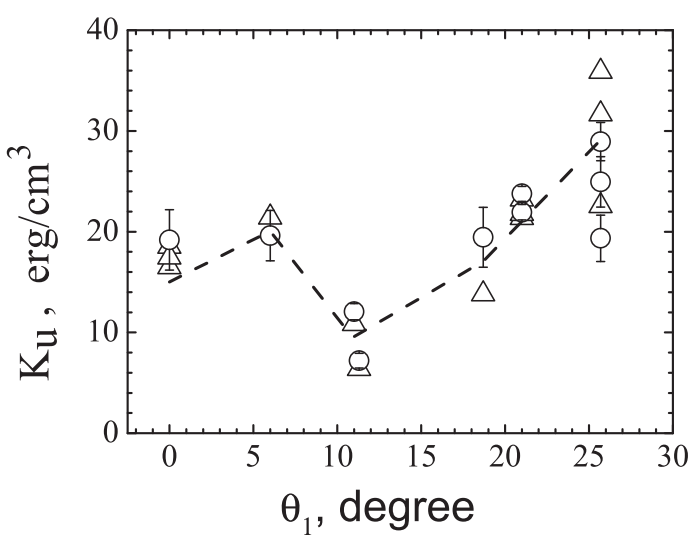

FIG. 4. The dependence of uniaxial anisotropy constant on the tilt angle of the (110)NGO substrate plane: open circles are FMR data at a frequency of 9.61 $\mathrm{GHz}$ and triangular are microwave absorption data at a frequency of 290.6 MHz. Dashed line connects small solid circles calculated using Eqs. (4) and (5) with the parameters taken from Table II.
Here, the first term describes the Zeeman energy, the second term describes the anisotropy energy with demagnetizing tensor $\hat{N}$, and the last term describes magnetocrystalline energy.

If taking into account only second order magnetocrystalline energy terms (it is valid only for predominant uniaxial anisotropy), the expression for $F_{m c}$ can be written in the following general form:

$$
F_{m c}=K_{x^{\prime}} \cdot \cos ^{2} \alpha_{x^{\prime}}+K_{y^{\prime}} \cdot \cos ^{2} \alpha_{y^{\prime}}+K_{z^{\prime}} \cdot \cos ^{2} \alpha_{z^{\prime}},
$$

where $K_{x^{\prime}}, K_{y^{\prime}}$, and $K_{z^{\prime}}$ are magnetocrystalline anisotropy constants and $\cos \alpha_{x^{\prime}}, \cos \alpha_{y^{\prime}}$, and $\cos \alpha_{z^{\prime}}$ are the direction cosines of the magnetization vector with respect to the crystallographic axes of the film. If the crystal structure is tilted to an angle $\theta_{1}$ around axis $x$ (see Fig. 1(a) for TB only) with respect to the film surface, Eq. (3) will be transformed and in coordinate system $(x, y, z)$ related to the film plane will be take the form

$$
F=-(\mathbf{M} \cdot \mathbf{H})+\left\{K_{\mathrm{y}^{\prime}}-K_{\mathrm{x}^{\prime}}+\left(K_{\mathrm{x}^{\prime}}-K_{\mathrm{z}^{\prime}}\right) \sin ^{2} \theta_{1}\right\} \cos ^{2} \alpha,
$$

which is equivalent to the case of a uniaxial magnetic anisotropy with the anisotropy constant

$$
K_{\mathrm{u}}=K_{\mathrm{y}^{\prime}}-K_{\mathrm{x}^{\prime}}+\left(K_{\mathrm{x}^{\prime}}-K_{\mathrm{z}^{\prime}}\right) \sin ^{2} \theta_{1} .
$$

Here, we note that this expression describes the experimental data well for the angular dependence of the anisotropy of $\mathrm{Fe}$ films deposited onto silver substrates having various tilt angles. $^{36,37}$

Dashed line in Fig. 4 connects the data points (solid circles) that were calculated using Eqs. (4) and (5) with three fitting parameters, $K_{x^{\prime}}, K_{y^{\prime}}$, and $K_{z^{\prime}}$. The values of these anisotropy constants are strongly dependent on strain emerged for corresponding directions. While the strains along $\mathrm{x}^{\prime}$ and $\mathrm{y}^{\prime}$ axes for small miscut angles are mainly fixed by substratefilm lattice mismatch, the strain along $\mathrm{z}^{\prime}$ axis can be varied due to relaxation or surface morphology. Therefore, we assumed that anisotropy constants $K_{x^{\prime}}$ and $K_{y^{\prime}}$ are independent of the angle $\theta_{1}$ and that constant $K_{z^{\prime}}$ is proportional to the crystalline strain along the [001] LSMO direction. The strain was determined as the difference between the experimental values of $\mathrm{a}_{\perp}$, taken from Table $\mathrm{I}$, and the lattice parameter of LSMO in the pseudocubic approximation $\left(a_{\mathrm{L}}=0.3876 \mathrm{~nm}\right.$ Ref. 3). It is seen that the dashed line in Fig. 4 describes the experimental points well enough; hence, we believe that analytical expressions (4) and (5) satisfactorily describe the real situation. Remarkably, the magnetic measurements unexpectedly have shown that $K_{\mathrm{u}}\left(6^{\circ}\right)>K_{\mathrm{u}}\left(10.9^{\circ}\right)$ and the calculations by Eq. (5) have repeated the same behaviour in spite $K_{\mathrm{u}}$ measurements and calculations by Eq. (5) rely on different measurement techniques.

\section{Magnetic anisotropy in bicrystal junctions}

The crystallographic misorientation of two parts of bicrystal substrate causes a change in the direction of the easy magnetization axis. The angular dependence of the FMR spectra strictly indicates the magnetic easy axis orientation 
for the epitaxial films. Films with the bridges have the parts with different forms (see Fig. 1(b)). This is the reason of arising of additional resonance lines. Nevertheless, it is always possible to identify the main doublet of lines and to trace their evolution during the rotation of the sample.

Fig. 5 shows an example of the angular dependence of FMR resonance field lines position corresponding to the two parts of the LSMO film separated by a $90^{\circ}$ RB-boundary. First, we note the dominant contribution of the uniaxial magnetic anisotropy over the cubic one, typical for LSMO/NGO (see part 2 and Refs. 7, 15, 30, and 38). For certain angle value, the experimental points are unavailable due to loss of second resonance line in the spectrum. We can conclude that the easy axes of magnetization of the film on each side of the bicrystal boundary are turned to each other by the angle of approximately $90^{\circ}$, the same as the angle of crystallographic misorientation.

Figure 6 shows the angular dependence of the absorption signal of the electromagnetic radiation at the room temperature at the frequency of $290.6 \mathrm{MHz}$ for the RB-type LSMO boundary with $2 \theta^{\prime}=90^{\circ}$. For simplicity, only the positive range of the external magnetic field is shown in Fig. 6. The change of the magnetic field to the opposite direction leads to the similar dependence. The narrow absorption lines recorded by this method are more reliable as compared to FMR and give possibility to detect the signals from the two parts of bicrystal boundary separately, whereas FMR method is unusable. This is particularly important for small misorientation angles of crystallographic axes, when the relatively large width of the FMR signals does not allow resolving the resonance lines from the two parts of the film.

It is can be clearly seen from Fig. 6 that there are two sets of absorption lines, which are attributed to the two parts of the bicrystal boundary. The first has a maximum at $H_{d c}=87 \mathrm{Oe}$ and the second at $H_{d c}=114 \mathrm{Oe}$. As noted above, these angles indicate the direction of the hard axes. The magnetic field values corresponding to the absorption maxima are equal to the uniaxial anisotropy fields. Using the fact that the hard and the easy axes of magnetization in a uniaxial ferromagnetic are perpendicular to each other, both measurement procedures provided the similar values for the parameters of the magnetic

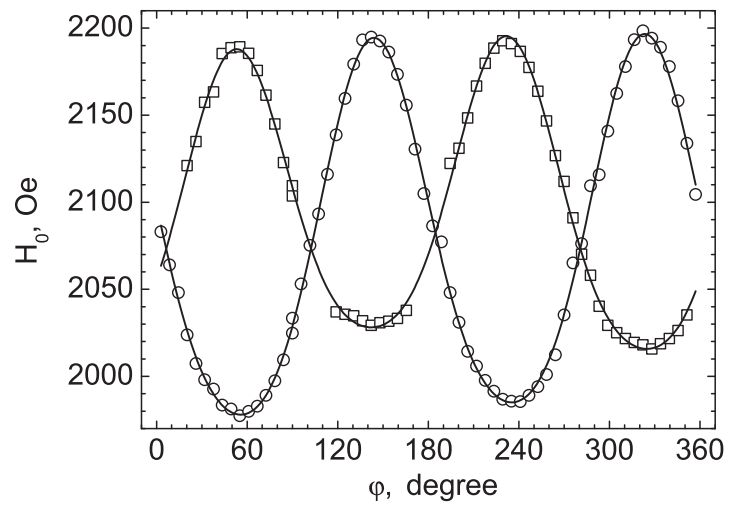

FIG. 5. The angular dependence of FMR resonance magnetic field for two lines observed in LSMO RB-boundary with misorientation angle $2 \theta^{\prime}=90^{\circ}$ at $\mathrm{T}=300 \mathrm{~K}$. Easy axis orientation for one part of bicrystal film corresponds to angles $\varphi \approx 60^{\circ} / 240^{\circ}$ (circles) and $\varphi \approx 150^{\circ} / 330^{\circ}$ for other part (squares). The DC magnetic field is along of RB-boundary at $\varphi=90^{\circ}$.

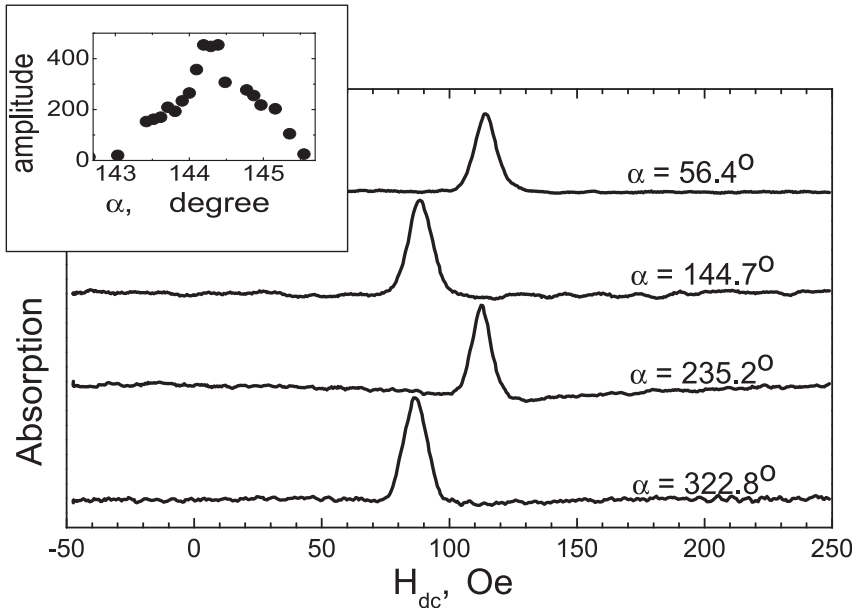

FIG. 6. Magnetic field dependence of the absorption signals at $290.6 \mathrm{MHz}$ for the RB-boundary with misorientation angle of $2 \theta^{\prime}=90^{\circ}$ for different values of the angle $\alpha$ between the external magnetic field and the axis $\mathrm{x}$, $\mathrm{T}=300 \mathrm{~K}$. The angular dependence of the absorption maximum (amplitude of the resonance signal) close the angle $\alpha=144^{\circ}$ is shown in the inset.

anisotropy in the bicrystal sample under study (see Table III). The presence in samples of the cubic magnetic anisotropy shifts the real peaks from the $\mathrm{H}_{\mathrm{u}}$ value in the second method that is the result of the numeric calculations. However, for films grown on NGO substrates, this shift is negligible, and it is easily taken into account in the calculations of magnetic parameters from the experimental data. It should be noted that the peak of susceptibility disappears in the second method if $\mathrm{H}_{\mathrm{c}}$ is about or more of $\mathrm{H}_{\mathrm{u}}$.

Previous magnetic measurements ${ }^{29-31}$ showed that the easy axis of the LSMO film deposited on (110) NGO coincides with the direction [11 0 ] NGO. As a result, for the RBboundaries with the misorientation angle for the directions [11 0$]$ NGO $2 \theta^{\prime}=90^{\circ}$, the misorientation of the easy axis is equal to $89^{\circ}-92^{\circ}$. As for TB-boundary which fabricates by rotation of (110) NGO plane around [110] NGO, the easy axes have to hold its directions. Nevertheless, we recorded the small deflection (about $1^{\circ}$ ) from the boundary direction that could be explained by the small crystal misorientation during bicrystal substrate fabrication.

TABLE III. Magnetic anisotropy of LSMO bicrystal junctions for $\mathrm{T}=300 \mathrm{~K}$

\begin{tabular}{lcccccc}
\hline \hline Structure type & $\begin{array}{c}2 \theta \\
(\mathrm{deg})^{\mathrm{a}}\end{array}$ & $\begin{array}{c}2 \theta^{\prime} \\
(\mathrm{deg})^{\mathrm{b}}\end{array}$ & $\begin{array}{c}H_{\mathrm{u} 1} / H_{\mathrm{u} 2} \\
(\mathrm{Oe})^{\mathrm{c}}\end{array}$ & $\begin{array}{c}\alpha_{\text {easy1 } 1} / \alpha_{\text {easy2 } 2} \\
(\mathrm{deg})^{\mathrm{d}}\end{array}$ & $\begin{array}{c}\alpha_{\text {hard1 }} / \alpha_{\text {hard2 }} \\
(\mathrm{deg})^{\mathrm{e}}\end{array}$ & $\begin{array}{c}\Delta \alpha \\
(\mathrm{deg})\end{array}$ \\
\hline RB-boundary & 0 & 90 & $123 / 98.4$ & $53.8 /-37.6$ & $146.4 / 54.3$ & 91.7 \\
TB-junction & 12 & 0 & $90 / 137$ & - & $89.4 / 90.6$ & 1.2 \\
RB-junction & 0 & 90 & $154 / 248$ & $48.7 /-40.9$ & $-47.4 / 44.0$ & 90.5 \\
\hline
\end{tabular}

${ }^{2} 2 \theta$ is the misorientation angle of the (001) LSMO/(110) NGO planes.

${ }^{\mathrm{b}} 2 \theta^{\prime}$ is the misorientation angle of [100] LSMO/[110] NGO directions.

${ }^{\mathrm{c}} \mathrm{H}_{\mathrm{u} 1, \mathrm{u} 2}$ are the magnetic anisotropy fields for the two parts of the bicrystal junction.

${ }^{\mathrm{d}} \alpha_{\text {easy } 1, \text { easy } 2}$ are the angles for the easy axis determined by FMR technique for the two parts of the bicrystal junction.

${ }^{\mathrm{e}} \alpha_{\text {hard } 1 \text {,hard } 2}$ are the angles for the hard axis obtained from microwave absorption technique at $300 \mathrm{MHz}$ for the two parts of the bicrystal junction.

${ }^{\mathrm{f}} \Delta \alpha$ is the total relative angle of the in-plane magnetizations of the two parts of the bicrystal junction. 


\section{MAGNETORESISTANCE OF BICRYSTAL JUNCTIONS}

\section{A. Temperature dependence of resistance}

Figure 7 shows the temperature dependence of the resistance of LCMO and LSMO TB-junctions obtained in the absence of an external magnetic field. The transition from the paramagnetic to the ferromagnetic state of manganites near the Curie temperature $T_{C}$ usually is accompanied by an insulator-metal transition, which manifests itself as a peak (or change curvature) in the temperature dependence of the resistance at $T_{P} . T_{P}$ is usually a few degrees below $T_{C}{ }^{1,29}$ The Curie temperature for bulk single-crystal and epitaxial films are equal to $T_{C} \approx 250 \mathrm{~K}$ and $T_{C} \approx 350 \mathrm{~K}$ for LCMO and LSMO, respectively. As can be seen from Fig. 7, for the bicrystal junctions we have $T_{P}=200 \mathrm{~K}$ and $T_{P}>300 \mathrm{~K}$ for LCMO and LSMO TB-junctions, respectively. But an additional peak in $R(T)$ for LCMO TB-junction at $T=130 \mathrm{~K}$ is observed. A comparison of the temperature dependence of the resistance of the TB-junction with the same size LCMO film bridge without boundary shows that the peak of resistance near $130 \mathrm{~K}$ is related to the TB-boundary. This indicates the presence of some part of the film with low $T_{p}$, whereas the main part of the films forming the TB-junction has a peak at $T_{P}=200 \mathrm{~K} .^{9,28}$ The presence of such an interfacial ferromagnetic layer with a lower $T_{C}$ layer is likely due to strong depletion of charge carriers in the boundary region as has been previously reported. ${ }^{39}$ There is no clear evidence of any other peak in the $R(T)$ dependence for LSMO bicrystal junctions which indicates negligible contribution of the interface layer with depressed ferromagnetism near the bicrystal boundary on the overall resistance. However, it should be noted that the detailed measurements of the temperature dependence of resistance in bicrystal junctions on STO carried out in Ref. 24 showed the presence of a boundary layer in LSMO interface with lowered value of the Curie temperature $\left(T_{P} \approx 250 \mathrm{~K}\right)$. The characteristic resistance of LCMO TBjunction was $R A=3 \cdot 10^{-6} \Omega \cdot \mathrm{cm}^{2}$ ( $R$ and $A$ are resistance and cross-sectional area of bicrystal junction, respectively) at $T=4.2 \mathrm{~K}$. This indicates that the bicrystal boundary in addition to ferromagnetic layer with suppressed $T_{C}$ also forms a potential barrier and transparency of this barrier can be roughly estimated as $10^{-3}-10^{-4}$. The lower characteristic

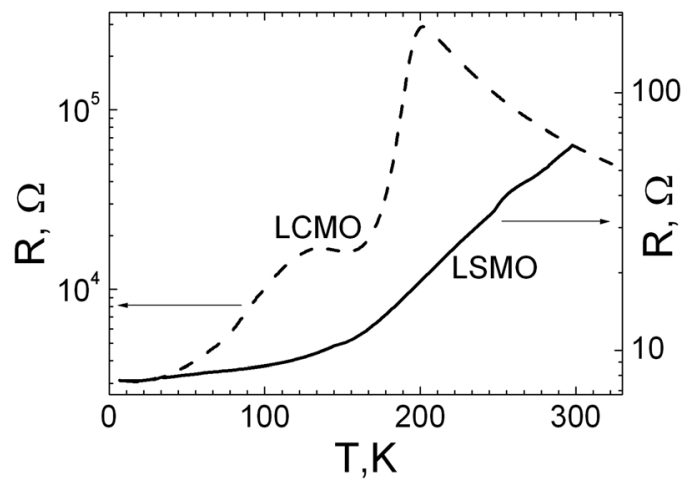

FIG. 7. Temperature dependence of the resistances for LSMO RB-junction $\left(2 \theta^{\prime}=90^{\circ}\right)\left(\right.$ solid line) and LCMO $\left(2 \theta=28^{\circ}\right)$ TB-junction (dashed line). The measurements were carried out at zero external field. resistance of the LSMO junctions compared to LCMO indicates that the potential barrier layer has a higher transparency than in the LSMO junctions.

\section{B. Magnetoresistance of bicrystal junctions}

Magnetoresistance of the LSMO TB-junction measured at four different temperatures is shown in Fig. 8. The figure demonstrates that the dependencies of magnetoresistance have prominent hysteresis loops, and at higher fields, the resistance decreases with increasing external magnetic field. The high-field part of the magnetoresistance in manganites is usually considered to be due to the effect of CMR and its contribution decreases with decreasing temperature. Contribution of anisotropic magnetoresistance also should be taken into account. ${ }^{40}$

Typically, the magnetoresistance for the junctions is defined as $M R=\left(R_{\max }-R_{0}\right) / R_{0}$, where $R_{\max }$ is the maximum resistance that usually correspond to antiparallel orientation of the magnetizations and $\mathrm{R}_{0}$ is the junction resistance at $H=0 \mathrm{Oe} .{ }^{40}$ Since in our case the resistance of the junction at zero field is quite high and obviously does not correspond to parallel orientation of magnetizations in the junction, the above definition would be lacking of informativity and not reflect the real situation with magnetoresistance behaviour. The presence of domain wall is not excluded also. In this paper, as a measure of the magnetoresistance, the following expression was chosen $M R^{\prime}=\left(R_{\max }-R_{H}\right) / R_{H}$, where $\mathrm{R}_{\mathrm{H}}$ is junction resistance at $H=0.75 \mathrm{kOe}$, where the magnetic hysteresis of $\mathrm{R}(\mathrm{H})$ is disappeared at helium temperatures.

Figure 9 shows the dependence of $M R^{\prime}$ on temperature, which in contrast to the $M R(T)$ (see inset Fig. 9), increases monotonically with decreasing temperature. In our definition of the magnetoresistance, the contribution of the CMR will be also included, but it can be neglected at such small magnetic fields especially at low temperatures.

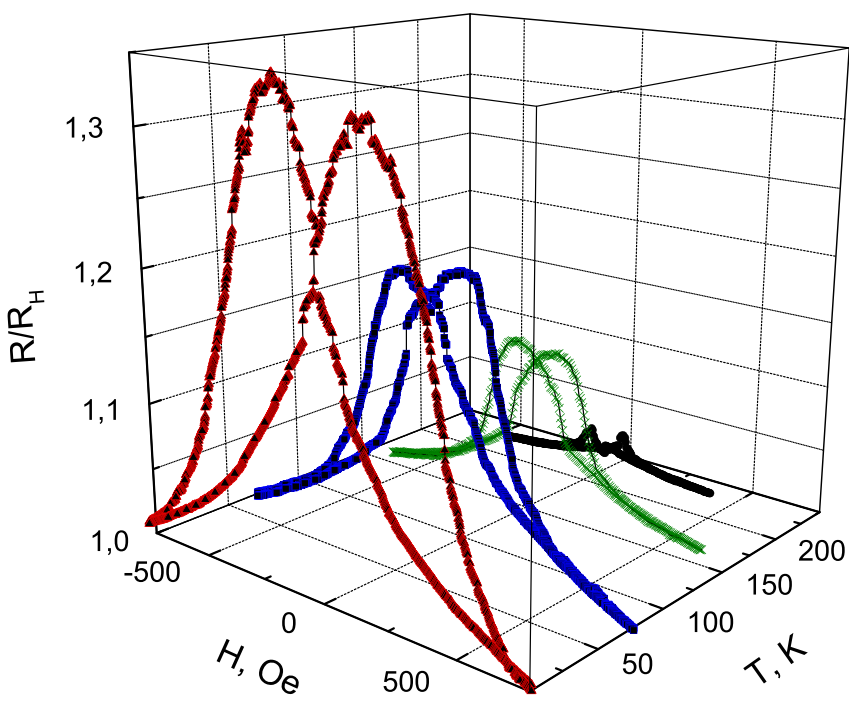

FIG. 8. Magnetoresistance of LSMO TB-junction with misorientation angle $2 \theta=38^{\circ}$ normalized on the resistance at $H=750 \mathrm{Oe}\left(R_{\mathrm{H}}\right)$ taken at four temperatures. Two curves for fixed temperature correspond to increasing and decreasing magnetic field. Magnetic field direction is determined by the angles $\alpha=45^{\circ}, \beta=90^{\circ}$ (see Fig. 1). 


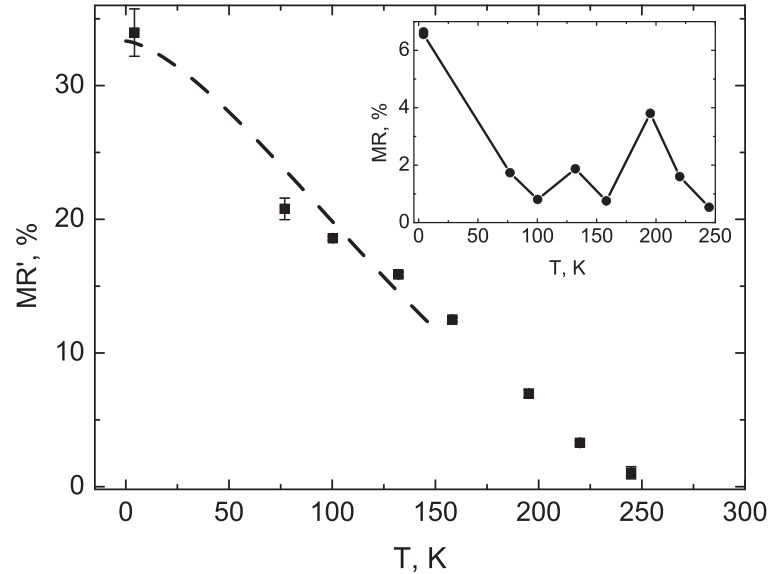

FIG. 9. Temperature dependence of the magnetoresistance $\mathrm{MR}^{\prime}$ (filled squares) for LSMO TB-junction. The dashed line represents the calculated temperature dependence of $\mathrm{MR}^{\prime}$ by Eqs. (8) and (10). The dependence of MR on temperature for the same junction $\left(2 \theta=38^{\circ}\right)$ is given in the inset.

To estimate the contribution of spin-polarized carriers to the bicrystal junction conductivity, we used the approach proposed in Refs. 41 and 42. We consider the conductance of spin-polarized carriers between two ferromagnetic separated by a tunneling barrier. It is necessary to take into account that the magnetization on both sides of bicrystal junction that are directed at different angles $\alpha_{1}$ and $\alpha_{2}$ with respect to the boundary. An expression for the spindependant part of conductivity $G_{\mathrm{sp}}$ in the situation is as follows: ${ }^{41-44}$

$$
G_{s p}=G_{s p}^{0}\left[1+P^{2} \cos \left(\alpha_{1}-\alpha_{2}\right)\right]
$$

Here, $G_{s p}^{0}$ is the conductivity at $\alpha_{1}-\alpha_{2}=\pi / 2$ and $\mathrm{P}$ is the polarization of the spins. Taking into account the contribution to the conductivity of non-polarized carriers $G_{\mathrm{ns}}$, we can write the expression for the resistance of the bicrystal junction as follows: ${ }^{43,44}$

$$
R=\frac{1}{G_{s p}+G_{n s}}=\frac{R_{s p}}{1+P^{2} \cos \left(\alpha_{1}-\alpha_{2}\right)+g},
$$

where $R_{s p}=1 / G_{s p}^{0}$ and $g=G_{n s} / G_{s p}^{0}$.

Our measurements of TB-junctions by the methods based on resonant absorption of electromagnetic radiation showed that misorientation of easy axes of the two parts of the junction is quite small (see Table III). For a rough estimate of $M R^{\prime}$, we assume that the point where magnetoresistance reaches its maximum corresponds to antiparallel orientation of magnetizations $\boldsymbol{M}_{1}$ and $\boldsymbol{M}_{2}$ (see Eq. (7)). It is possible when the magnitude of external magnetic field is between two values of $\mathrm{H}_{\mathrm{u}}$ for two parts of the junction (Table III). For sufficiently large values of the external magnetic field, the magnetizations are parallel to each other and directed along the external field. It should be noted that the angle between $\mathrm{M}_{1}$ and $\mathrm{M}_{2}$ may differ slightly from $180^{\circ}$, but we suppose this difference is small enough. We also assume $\mathrm{P}=100 \%$ at low $\mathrm{T}$ that was confirmed by experiments and theoretical estimations (see reviews Refs. 1-5) and the measurements. ${ }^{43-45}$ The above presentation of magnetic junction resistance allows us to estimate the ratio between conductances of spin-polarized and non-polarized carriers. Using Eq. (7), the maximum magnetoresistance is equal to $R_{\max }=R_{s p} /\left(1-P^{2}+g\right)$ ). For large fields, when the magnetizations of $\boldsymbol{M}_{1}$ and $\boldsymbol{M}_{2}$ are parallel, the magnetoresistance is equal to $R_{H}=R_{s p} /\left(1+P^{2}+g\right)$.

We estimate $g$ from $M R^{\prime}$

$$
M R^{\prime}=\left(R_{\max }-R_{H}\right) / R_{H}=2 P^{2} /\left(1-P^{2}+g\right) .
$$

Substituting $\mathrm{P}=1$ in Eq. (8), which is corresponding to $100 \%$ magnetic polarization, we obtain

$$
M R^{\prime}=2 / g
$$

From the data presented in Figure 9, we obtain $g=5.8 \pm 0.4$. Consequently, the measured DC conductivity of the junction at small magnetic field is mainly determined by the transfer of non-polarized carriers. The temperature dependence of the polarization has a power-law form ${ }^{43,45}$

$$
P(T)=P_{0}\left(1-\varepsilon T^{3 / 2}\right) .
$$

Substituting Eq. (10) into Eq. (8) and fitting with experiment, we get $\varepsilon=2 \cdot 10^{-4} \mathrm{~K}^{-3 / 2}$. The obtained value of $\varepsilon$ is in the order of magnitude equal to those obtained by using photoemission spectroscopy of free surface of the LSMO films $\varepsilon=4 \cdot 10^{-4} \mathrm{~K}^{-3 / 2} \cdot{ }^{45}$ But it is almost one order of magnitude higher than for magnetic tunnel structures based on the LSMO films with STO tunnel barrier $\left(\varepsilon=4 \cdot 10^{-5} \mathrm{~K}^{-3 / 2}\right){ }^{45,46}$ We note that TB-junctions with a smaller angle misorientation $\left(2 \theta=12^{\circ}\right)$ show smaller value of the magnetoresistance (by a few percent) and a reduced characteristic resistance RA. As a consequence, we conclude that the portion of nonpolarized carriers determining the junction resistance increases with decreasing of misorientation angle. Note that the magnetoresistance is considerably higher in the LCMO TB-junctions, where there is a transition layer with a lower Curie temperature in vicinity of bicrystal boundary and the characteristic boundary resistance is greater than for LSMO bicrystal junction. ${ }^{28}$ But we do not think that the suppression of $\mathrm{T}_{\mathrm{C}}$ is the reason for the resistance variation.

\section{DC voltage dependence of the conductivity}

To study the mechanism of charge and spin transport, the conductivity of the TB-junctions as function of the DC applied voltage was measured in a temperature range from 4.2 to $300 \mathrm{~K}$. Electron transport has been described by the mechanism of elastic tunneling through a rectangular barrier. $^{47,48}$ In this model, the dependence of the junction resistance on the magnetic field is absent, and the change in the junction conductivity occurs due to the variation of the barrier shape in the presence of voltage on the junction. Voltage dependence has the following form: $G(V)=G_{0}+G_{2}\left|\mathrm{~V}^{2}\right|$, where of $G_{0} \gg G_{2}\left|\mathrm{~V}^{2}\right|$. But this model is not applicable here since the $G(V)$ measured for bicrystal junction does not follow the law of $V^{2}$ in full range of $V$.

A further development of the previously described model takes into account the presence in the vicinity of the bicrystal boundary of the interface layers with specific 
electrophysical characteristics. The properties of this interface layer differ significantly from the properties of the electrodes due to additional scattering centers and the shorter mean free path. The clearest evidence for the existence of the interface layer with different characteristic properties is observed in the LCMO RB-junctions. ${ }^{28}$ In the layer with short mean free path, the electron-electron (e-e) interaction may increase due to the weak localization effect. ${ }^{49,50}$ In our case, strong e-e interaction is a particular characteristic of manganites ${ }^{1}$ leading to a conductance of the form: $G(V, T)=G_{0}+G_{0.5}\left|\mathrm{~V}^{0.5}\right|$. The $G_{0}$ could depend on $H$ due to the quantum corrections in conductivity of the layer in nonmagnetic material. ${ }^{49,50}$ The term $G_{0.5}\left|V^{0.5}\right|$ decreases rapidly with increasing temperature as observed in the disordered metal oxides ${ }^{50}$ at temperatures up to $10 \mathrm{~K}$. Indeed, in our experiment at low temperatures $(\mathrm{T}<18 \mathrm{~K})$, we can clearly distinguish a contribution proportional to $V^{0.5}$ in $G(V)$ (see Fig. 10). The conductivity mechanism for the junction containing localized states in the barrier was considered by Glazman and Matveev ${ }^{51}$ and predicts the temperature dependence of $G(T) \sim T^{4 / 3}$, which in our case was not observed.

In Refs. 52 and 53, the scattering of carriers on magnetic excitations is considered, which leads to a non-linear voltage dependence of the conductivity. The model of scattering of spin-polarized carriers ${ }^{54}$ suggests the dependence of $G(V)$ $=G_{0}+G_{2}\left|\mathrm{~V}^{2}\right|+G_{3 / 2}\left|\mathrm{~V}^{3 / 2}\right|$ for the conductivity of the magnetic junction. The term $G_{2}\left|V^{2}\right|$ reflects the influence of bulk magnons and $G_{3 / 2}\left|V^{3 / 2}\right|$ of surface antiferromagnetic magnons. By comparing our experimental data for $G(V)$ (see Fig. 10) with this model, we conclude that in the range of high temperatures $(T \geq 64 \mathrm{~K})$ the influence on spinscattering mechanism of surface antiferromagnetic magnons is dominant over bulk magnons.

Consequently, our analysis of the voltage dependence of the conductivity of the bicrystal junctions shows that two scattering mechanisms are important: the electron-electron interaction at low temperatures suggesting the presence of interface layers at the bicrystal boundary and the scattering of spin-polarized carriers by antiferromagnetic magnons at

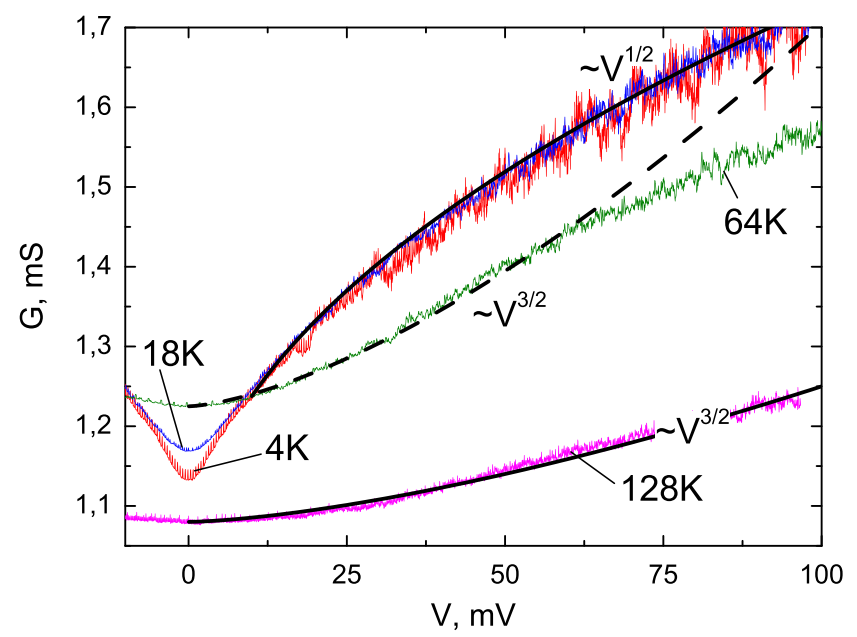

FIG. 10. DC voltage dependence of the conductance $G(V)$ of LSMO RBjunction $\left(2 \theta=38^{\circ}\right)$ at temperatures $\mathrm{T}=4 \mathrm{~K}, 18 \mathrm{~K}, 64 \mathrm{~K}$, and $128 \mathrm{~K}$. The lines show the fits of the experimental curves by different power functions. higher temperatures. ${ }^{54}$ The increase in the magnetoresistance with decreasing temperature occurs due to both the increase of the magnetic polarization and weakening of the spin scattering mechanism.

\section{CONCLUSION}

Microwave resonance methods based on FMR technique and RF absorption in the vicinity of uniaxial magnetic anisotropy field were used for investigation of magnetic anisotropy in epitaxial LSMO films and bicrystal junctions. The weak orthorhombicity of the NGO substrate leads to a domination of uniaxial magnetic anisotropy in the substrate surface plane. Measurements of the angular dependence of the ferromagnetic resonance magnetic field in the bicrystal junctions showed the presence of two ferromagnetic subsystems. For bicrystal boundaries with the basal plane rotation around normal to the substrate plane (RB-junction), the angles between the magnetic easy axes coincide with the crystallographic misorientation angles. On the other hand for bicrystal boundaries with the basal plane rotation around the bicrystal boundary line (TB-junction), the direction of the easy axes of magnetization were found along the bicrystal boundary. The magnetization misorientation practically did not depend on the angle of the crystallographic planes misorientation. The magnitude of the magnetoresistance $\left(\mathrm{MR}^{\prime}\right)$ for TB-junctions increases with decreasing temperature, but even at $\mathrm{T}=4.2 \mathrm{~K}$, when the polarization of the LSMO films is supposed to be closed to $100 \%, \mathrm{MR}^{\prime}$ is only $30 \%$. We showed that the low value of the magnetoresistance for the LSMO bicrystal junctions can be caused by two spin-flip scattering mechanisms: the strong electron-electron interactions in a disordered interface layer at the bicrystal boundary at low temperatures and the scattering by anti ferromagnetic magnons at high temperatures.

\section{ACKNOWLEDGMENTS}

The authors are grateful to V. A. Atsarkin, K. I. Constantinyan, A. Kalabukhov, A. A. Klimov, I. M. Kotelyanskii, V. A Luzanov, and S. A. Nikitov for useful discussion on the data and help with the measurements. This work was supported by the programs of the Russian Academy of Sciences, Russian Ministry of Education and Sciences, Leading Scientific School SSh-2456.2012.2, RFBR Project-11-02-01234a, 11-02-00349a, and 12-07$31207 \mathrm{~mol}$, and the Swedish Institute Visby project.

${ }^{1}$ Yu. A. Izyumov and Yu. N. Skryabin, Phys. Usp. 44, 109 (2001).

${ }^{2}$ W. Prellier, Ph. Lecoeur, and B. Mercey, J. Phys. Condens. Matter 13, R915 (2001).

${ }^{3}$ A.-M. Haghiri-Cosnet and J. P. Renard, J. Phys. D: Appl. Phys. 36, R127 (2003).

${ }^{4}$ M. Ziese, Rep. Prog. Phys. 65, 143-249 (2002).

${ }^{5}$ I. Zutic, Rev. Mod. Phys. 76, 323 (2004).

${ }^{6}$ A. Kadigrobov, Z. Ivanov, T. Claeson, R. I. Shekhter, and M. Jonson, Europhys. Lett. 67, 948 (2004).

${ }^{7}$ P. Dey, T. K. Nath, and A. Tarapher, Appl. Phys. Lett. 91, 012511 (2007).

${ }^{8}$ F. Tsui, M. C. Smoak, T. K. Nath, and C. B. Eom, Appl. Phys. Lett. 76, 2421 (2000).

${ }^{9}$ Y. P. Lee, S. Y. Park, Y. H. Hyun, J. B. Kim, V. G. Prokhorov, V. A. Komashko, and V. L. Svetchnikov, Phys. Rev. B 73, 224413 (2006). 
${ }^{10}$ Yan Wu, Y. Suzuki, U. Rudiger, J. Yu, A. D. Kent, T. K. Nath, and A. D. Eom, Appl. Phys. Lett. 75, 2295 (1999).

${ }^{11}$ M. Bibes, S. Valencia, L. Balcells, B. Martinez, J. Fontcuberta, M. Wojcik, S. Nadolski, and E. Jedryka, Phys. Rev. B 66, 134416 (2002).

${ }^{12}$ H. Y. Hwang, T. T. M. Palstra, S.-W. Cheong, and B. Batlogg, Phys. Rev. B 52, 15046 (1995).

${ }^{13}$ A. J. Millis, T. Darling, and A. Migliori, J. Appl. Phys. 83, 1588 (1998).

${ }^{14}$ M. C. Martin, G. Shirane, Y. Endoh, K. Hirota, Y. Moritomo, and Y. Tokura, Phys. Rev. B 53, 14285 (1996).

${ }^{15}$ H. Boschker, M. Mathews, E. P. Houwman, H. Nishikawa, A. Vailionis, G. Koster, G. Rijnders, and D. H. A. Blank, Phys. Rev. B 79, 214425 (2009).

${ }^{16}$ Z.-H. Wang, G. Cristiani, and H.-U. Habermeire, Appl. Phys. Lett. 82, 3731 (2003).

${ }^{17}$ M. Mathews, R. Jansen, G. Rijnders, J. C. Lodder, and D. H. A. Blank, Phys. Rev. B 80, 064408 (2009).

${ }^{18}$ K. Steenbeck and R. Hiergeist, Appl. Phys. Lett. 75, 1778 (1999); K. Steenbeck, R. Hiergeist, C. Dubourdiueu, and J. P. Senateur, ibid. 80 , 3361 (2002).

${ }^{19}$ M. Mathews, F. M. Postma, J. C. Lodder, R. Lansen, G. Rijnders, and D. H. A. Blank, Appl. Phys. Lett. 87, 242507 (2005).

${ }^{20}$ N. D. Mathur, G. Burnell, S. P. Isaac, T. J. Jackson, B.-S. Teo, J. L. MacManus-Driscoll, L. F. Cohen, J. E. Evetts, and M. G. Blamire, Nature 387, 266 (1997).

${ }^{21}$ J. Klein, C. Hofener, S. Uhlenbruck, L. Alff, B. Buchner, and R. Gross, Europhys. Lett. 47, 371 (1999)

${ }^{22}$ R. Gunnarsson and M. Hanson, Phys. Rev. B 73, 014435 (2006).

${ }^{23}$ J. B. Philipp, C. Hofener, S. Thienhaus, J. Klein, L. Alff, and R. Gross, Phys. Rev. B 62, R9248 (2000).

${ }^{24}$ S. P. Isaac, N. D. Mathur, J. T. Evetts, and M. G. Blamaire, Appl. Phys. Lett. 72, 2038 (1998).

${ }^{25}$ R. Werner, R. Petrov, A. Yu. Mino, L. Alvarez, R. Kleiner, D. Koelle, B. A. Davidson, Appl. Phys. Lett. 98, 162505 (2011).

${ }^{26}$ Y. Y. Divin, U. Poppe, C. L. Jia, P. M. Shadrin, and K. Urban, Physica C 372-376, 115 (2002).

${ }^{27}$ I. V. Borisenko, I. M. Kotelyanski, A. V. Shadrin, P. V. Komissinski, and G. A. Ovsyannikov, IEEE Trans. Appl. Supercond. 15, 165 (2005).

${ }^{28}$ I. V. Borisenko and G. A. Ovsyannikov, Phys. Solid State 51, 309 (2009).

${ }^{29}$ G. Alejandro, L. B. Steren, H. Pastoriza, D. Vega, M. Granada, J. C. Royas Sanchez, M. Sirena, and B. Alascio, J. Phys.: Condens. Matter 22, 346007 (2010).
${ }^{30}$ G. A. Ovsyannikov, A. M. Petrzhik, I. V. Borisenko, A. A. Klimov, Yu. A. Ignatov, V. V. Demidov, and S. A. Nikitov, JETP 108, 48 (2009).

${ }^{31}$ V. V. Demidov, I. V. Borisenko, A. A. Klimov, G. A. Ovsyannikov, A. M. Petrzhik, and S. A. Nikitov, JETP 112, 825 (2011).

${ }^{32}$ B. A. Belyaev, A. V. Izotov, and S. Ya. Kiparisov, JETP Lett. 74, 226 (2001).

${ }^{33}$ T. M. Vasilevskaya and D. I. Sementsov, JETP 110, 754 (2010).

${ }^{34}$ A. E. Mefed and V. V. Demidov, Instrum. Exp. Tech. 51, 418 (2008).

${ }^{35}$ S. V. Vonsovsky, Magnetism (Wiley, New York, 1974).

${ }^{36}$ R. K. Kawakami, E. J. Escorcia-Aparicio, and Z. Q. Qui, Phys. Rev. Lett. 77, 2570 (1996).

${ }^{37}$ Y. Z. Wu, C. Won, and Z. Q. Qui, Phys. Rev. B 65, 184419 (2002).

${ }^{38}$ N. G. Bebenin, R. I. Zainullina, V. V. Mashkautsan, V. V. Ustinov, and Ya. M. Mukovskii, Phys. Rev. B 69, 104434 (2004).

${ }^{39}$ R. Gunnarson, A. Kadigrobov, and Z. Ivanov, Phys. Rev. B 66, 024404 (2002).

${ }^{40}$ J. O’Donnel, M. Onellion, and M. S. Rzchowski, Phys. Rev. B 55, 5873 (1997).

${ }^{41}$ M. Julliere, Phys. Lett. A 54, 225 (1975).

${ }^{42}$ J. C. Slonczewski, Phys. Rev. B 39, 6995 (1989).

${ }^{43}$ C. H. Shang, J. Nowak, R. Jansen, and J. S. Moodera, Phys. Rev. B 58, R2917 (1998).

${ }^{44}$ R. Gunnarsson, M. Hansen, and C. Dobourdieu, J. Appl. Phys. 96, 482 (2004).

${ }^{45}$ J.-H. Park, E. Vescovo, H.-J. Kim, C. Kwon, R. Ramesh, and T. Venkatesan, Phys. Rev. Lett. 81, 1953 (1998).

${ }^{46}$ V. Garcia, M. Bibes, A. Barthelemy, M. Bowen, E. Jacquet, J.-P. Contour, and A. Fert, Phys. Rev. B 69, 052403 (2004).

${ }^{47}$ A. M. Petrzhik, V. Demidov, G. A. Ovsyannikov, I. V. Borisenko, and A. V. Shadrin, JETP 115, 876 (2012).

${ }^{48}$ W. Westerburg, F. Martin, S. Freiedrich, M. Maier, and G. Jakob, J. Appl. Phys. 86, 2173 (1999).

${ }^{49}$ P. A. Lee and T. V. Ramakrishnan, Rev. Mod. Phys. 57, 287 (1985).

${ }^{50}$ M. E. Gershenzon, V. N. Gubankov, and M. I. Faley, Sov. Phys. JETP 63, 1287 (1986).

${ }^{51}$ L. I. Glazman and K. A. Matveev, Sov. Phys. JETP 67, 1276 (1988).

${ }^{52}$ C. A. Dartora and G. G. Cabrera, J. Appl. Phys. 95, 6058 (2004).

${ }^{53}$ F. Guinea, Phys. Rev. B 58, 9212 (1998).

${ }^{54}$ N. Khare, U. P. Moharil, A. K. Gupta, A. K. Raychaudhuri, S. P. Pai, and R. Pinto, Appl. Phys. Lett. 81, 325 (2002). 